\title{
MICROTENSILE BOND STRENGTH OF A SELF-ETCH ADHESIVE USED AS A CARRIER FOR A REMINERALIZING AGENT
}

\author{
Mona M. Ghoneim * and Moustafa N. Aboushelib**
}

\begin{abstract}
Objectives: resin adhesive could be used as a carrier of nano-hyrdoxy apatite particles added to treat early enamel caries. The aim of this study was to evaluate microtensile bond strength of a remineralizing adhesive to early carious enamel lesion

Methods: an artificial caries model was used to induce early non cavitated enamel caries-like lesion in maxillay centrals. Nano hydroxyapatite particles (nHA) were applied in two forms to a self-etch resin adhesive: particles were directly added to resin adhesive or silanated before addition. Unmodified adhesive was used as control. Micro-tensile bond strength (MTBS) was performed to evaluate bond strength to demineralized enamel after application remineralizing resin adhesive $(n=20, \alpha=0.05)$. Scanning electron microscopy (SEM) was performed to assess mode of failure and to study adhesive enamel interface.
\end{abstract}

Results: Direct addition of nano-particles resulted in significant reduction $(\mathrm{F}=32, \mathrm{P}<0.001)$ in MTBS (12 MPa \pm 3.8 ) compared to addition of silanated particles (24 MPa \pm 3.2 ) which presented comparable values to the control group $(26 \mathrm{MPa} \pm 4.1)$. SEM image analysis revealed adhesive failure observed for directly added particles while the other two groups demonstrated cohesive failure observed in the resin adhesive. Direct addition of nHA resulted in partial remineralization of enamel ( $42 \%$ recovery in calcium content.

Conclusions: Self-etch resin adhesive could be used as a carrier for nano hydroxyapatite particles without compromising bond strength to early caries-like enamel lesions.

\section{INTRODUCTION}

Dental caries remains to be the most prevalent oral lesion. It is a worldwide spreading disease and continues to be one of the pandemics among children regardless of the socio-economic status and how developed a country is ${ }^{(1)}$. Dental caries is often not self-limiting and without proper care, caries can progress until the tooth is destroyed (2). For the last 150 years, traditional management of cavitated carious lesions in dental practice was mainly "Restorative" in nature. Carious tissue in enamel and dentin is excised and the space is filled by the restorative material of choice ${ }^{(3)}$.

\footnotetext{
* Associate Professor of Restorative Dentistry, Faculty of Dentistry, Alexandria University, Egypt.

** Professor of Biomaterials, Faculty of Dentistry, Alexandria University, Egypt.
} 
Epidemiological data on dental caries show that prevention and treatment needs have evolved significantly over the past two decades ${ }^{(4)}$. Prevention of dental caries has always been difficult to tackle. Dental professionals have actively participated in caries prevention through plaque removal and dental hygiene techniques; reinforcing the need for reduction in cariogenic refined carbohydrates ingestion; topical application of fluoridated dentifrices, rinses and gels; systemic water fluoridation; placement of pit and fissure sealants ${ }^{(5)}$. Local fluoride application in various forms and systemic fluoride delivery are the golden standards of caries prevention and have proven high efficiency in preventing and arresting caries worldwide $^{(6,7)}$. Unfortunately these methods are not efficient enough to completely prevent tooth decay ${ }^{(8)}$. Apparently fluoride alone can't totally eradicate caries incidence and additional strategies are needed ${ }^{(9)}$.

Research efforts focused basically on prevention of caries but less emphasis was directed towards remineralization of early carious lesions. Synthetic nano hydroxyapatite (nHA) is considered one of the most biocompatible and bioactive materials, and has gained wide acceptance in medicine and dentistry in recent years ${ }^{(10)}$. It mimics hydroxyapatite crystals in morphology and structure (11) and arrested mineral loss in vitro ${ }^{(12)}$. Recently several reports have shown that synthetic nano-hydroxyapatites has some potential to repair and remineralize early carious lesions in both enamel and dentin ${ }^{(10-15)}$.

The concept of self-etching approach was created approximately 20 years ago, however, the first and second generations of bonding agents can be considered self-etch materials because no acid etching/rinsing or conditioning step were used. Selfetch adhesive systems are considered simplified adhesive materials and they offer some advantages over conventional etch-and rinse systems ${ }^{(16)}$. Sadat et al showed that mixing of $0.2 \%-.5 \% \mathrm{nHA}$ with single bottle bonding agent as a filler material can improve mechanical properties of the bonding agent and bond strength to sound dentin ${ }^{(17)}$. Addition of nHA particles to conventional resin adhesives improved shear bond strength of orthodontic brackets to sound enamel ${ }^{(18)}$. There is consistent evidence that demineralization of the enamel surface reduces the bond strength of regular adhesive systems ${ }^{(19)}$. However, there is lack of evidence about bond strength to demineralized caries-like enamel lesions using nHA enriched resin adhesives. The aim of this study was to evaluate microtensile bond strength of a remineralizing self-etch adhesive enriched with two forms of nano-hydroxy apatite particles to early carious enamel. The proposed null hypothesis was that both application methods will perform similarly.

\section{MATERIALS AND METHODS}

Extracted teeth were collected and demineralized in-vitro to produce early enamel caries-like lesions. Produced lesions were characterized by scanning electron microscopy and cross-sectional hardness testing; Nano-Hydroxyapatite particles were partially hydrolyzed and added to self-etch resin adhesive before and after silanation. Subsequently composite blocks were bonded to the enamel surface and microtensile bond strength was evaluated.

\section{Production of caries-like lesions}

A total of 55 extracted caries free human maxillary central incisors were selected. Teeth were sectioned at cemento-enamel junction level using diamond disc mounted on a precision cutter (Micracut 1.5, Metkon, Turkey). The cut crowns were covered with 2 layers of acid resistant varnish leaving only $4 \times 4 \mathrm{~mm}$ facial window. Demineralizing solution was prepared according to Ten Cate and Djustiers ${ }^{(20)}$. Briefly the solution was composed of $50 \mathrm{mM}$ acetic acid $+2.2 \mathrm{mM} \mathrm{Ca}\left(\mathrm{NO}_{3}\right)_{2} \cdot 2 \mathrm{H}_{2} \mathrm{O}+$ $2.2 \mathrm{mM} \mathrm{KH}_{2} \mathrm{PO}_{4}+0.1 \mathrm{ppm} \mathrm{NaF}$. The $\mathrm{pH}$ of the solution was adjusted to 4.2 using small amounts of 
$\mathrm{NaOH}, \mathrm{pH}$ was measured daily by $\mathrm{pH}$ meter (Jenway $3020 \mathrm{pH}$ meter, UK) and $\mathrm{pH}$ was kept between 4.2 and 4.25 by adding refreshening amounts of HCL $30 \%$ solution. Teeth were immersed in the demineralizing solution for 3 days. Solution was freshened daily.

\section{Cross-sectional micro hardness test}

10 Specimens were randomly selected and longitudinally sectioned using a diamond disc mounted in a precision cutter (Micracut 1.5, Metkon, Turkey). Sections then were embedded in acrylic resin blocks exposing the cross section. Cross-section micro-hardness was performed using a Vickers indentation point (Wilbert Wilson, Germany) using $200 \mathrm{~g}$ weight applied for 30seconds. Measurements were taken at from the surface of enamel to a maximum depth of $500 \mathrm{um}$ at 25 um steps. Hardness values of sound enamel was measured and used as a control. These tests were repeated after 3 months of water storage to measure remineralization potential of each test group.

\section{Assessment of morphological changes and cal- cium loss}

Carious enamel specimens were gold-sputter coated (Fine coat jfc-1100E, Jeol, Munich, Germany) and examined using scanning electron microscope (JSM-5300, Jeol, Munich, Germany). Calcium and phosphorus content were evaluated from the surface of carious enamel to a maximum depth of 300 um at 25 um steps using Energydispersive X-ray spectroscopy (EDX) unit and average spot size of $1 \mathrm{um}$.

\section{Preparation of nano- hydroxyapatite delivery forms (nHA)}

Nano hydroxyapatite particles were prepared by sol gel chemical reaction. Particle size and distribution were evaluated using particle size analyzer and particles size in range of 40-60 nanometers was selected. The particles were paritially hydrolyzed using phosphoric acid $10 \%$ wt nHA powder was either directly mixed with a onestep self-etch resin adhesive (Single bond, 3M ESPE, St Paul, MN, USA) or coated with a silane coupling agent before addition to the resin adhesive. A coat of enriched self-etch resin adhesive was applied and polymerized following manufacturer recommendations. Resin composite (Filteck z350, 3M ESPE, USA) were added and polymerized on the demineralized facial surface.

\section{Microtensile bond strength test (MTBS)}

Microbars were prepared by cutting bonded specimens using precision cutting machine (Isomet 1000, Buehler, Lake Bluff, Ill, USA). 25 microbars, $1 \times 1 \times 5 \mathrm{~mm}$, were chosen randomly from cut specimens. All specimens were loaded to failure using universal testing machine (Instron 6022, Instron Limited, High Wycombe, UK) at constant crosshead speed of $0.5 \mathrm{~mm} / \mathrm{min}$. Fractured microbars were examined under stereo-microscope (SZ 11, Olympus Inc, Osaka, Japan) to detect failure mode.

\section{Scanning electron microscopy (SEM)}

Intact sections were collected during sectioning procedure as described previously. The sections were polished using silicon carbide paper $(800,1000$, and 1200 grit). The sections were ultrasonically cleaned in demineralized water for $15 \mathrm{~min}$ and dried at $60^{\circ} \mathrm{C}$ for $60 \mathrm{~min}$. Sections were gold sputter coated and prepared for SEM examination (XL 30, Phillips, Eindhoven, the Netherlands).

Levene's test of equality of error variances was performed to test the null hypothesis that error variance in MTBS was similar in tested groups. One way analysis of variance (ANOVA) was selected to analyze the data with 1 within-group factor (method of application of nHA). Bonferroni post hoc test was selected for pair-wise comparisons $(a=.05)$. Data were analyzed using computer software (SPSS 14.0; SPSS, Inc, Chicago, Ill). 


\section{RESULTS}

Cross sectional hardness values showed significant differences between demineralized enamel lesions and sound enamel $(\mathrm{F}=35, P<.008)$. Mean lesion depth was $377.1 \pm 47.5 \mu \mathrm{m}$ after which hardness values reached a constant plateau equivalent of sound enamel, Figure 1. Mean hardness value of demineralized enamel was 202.6 HVN compared to $380.3 \mathrm{HVN}$ of sound enamel. Scanning electron microscopy revealed characteristic inter-rod dissolution and exposure of enamel rod peripheries resulting in increasing apparent surface roughness, Figure 2.

EDX demonstrated that demineralized enamel had a significant reduction of calcium and phosphorous content compared to sound enamel $(\mathrm{F}=33, P<.005)$. There was a marked reduction of $54 \% \mathrm{wt}$ of both calcium and phosphorous content within the observed lesion. Calcium and phosphorous content reached normal values beyond the observable mean lesion depth. 3 months of water storage resulted in $42 \%$ recovery of calcium content for the directly added nano-particles. This was associated with increase of enamel microhardness, 255 VHN. Silanated particles failed to produce similar results.

There were significant statistical differences in MTBS values between the tested groups. Direct addition of hydroxyapatite particles reduced $(\mathrm{F}=32$, $\mathrm{P}<0.001)$ MTBS values $(12 \mathrm{MPa} \pm 3.8)$ compared non-modified self-etch adhesive $(26 \mathrm{MPa} \pm 4.1)$. On the contrary application of silanated nano particles did not influence MTBS values (24 MPa \pm 3.2) compared to the unmodified adhesive. SEM of fractured microbars obtained from different groups showed that direct addition of nHA resulted in agglomeration of the particles on the on the surface of demineralized enamel.. On the other hand, using silanated particles did not result in creation of structural defects on the surface of demineralized enamel and remained properly distributed in the resin adhesive, Figure 3 and 4.

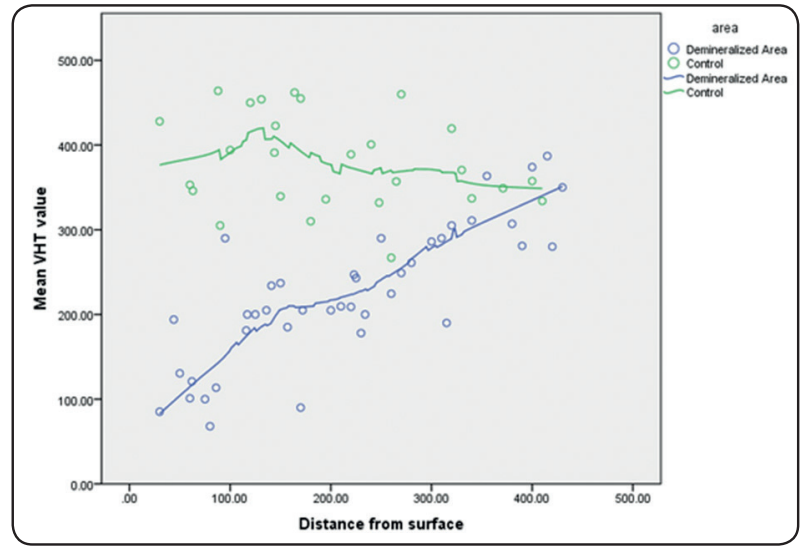

Fig. (1) Mean micro-hardness value of demineralized and sound enamel and distance from the tooth surface.

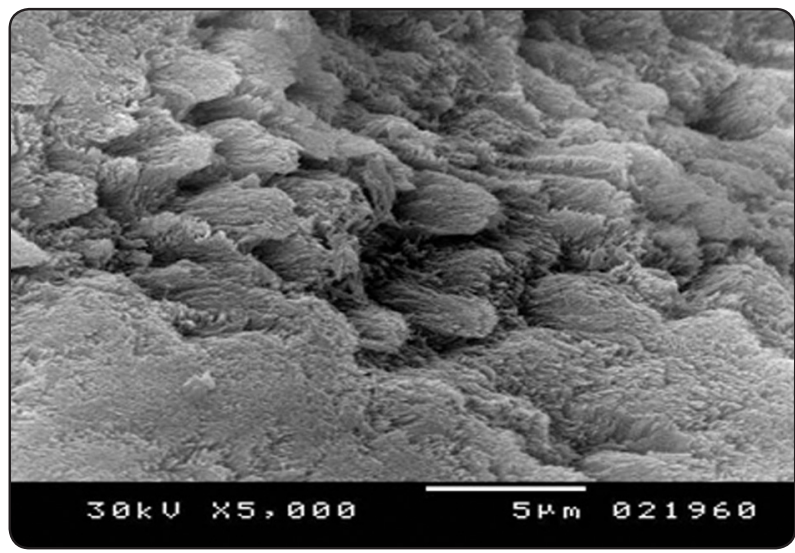

Fig. (2) Scanning electron microscope, 5000x, for in-vitro demineralized enamel surface showing characteristic dissolution of the inter-rod enamel with more preserved rod core.

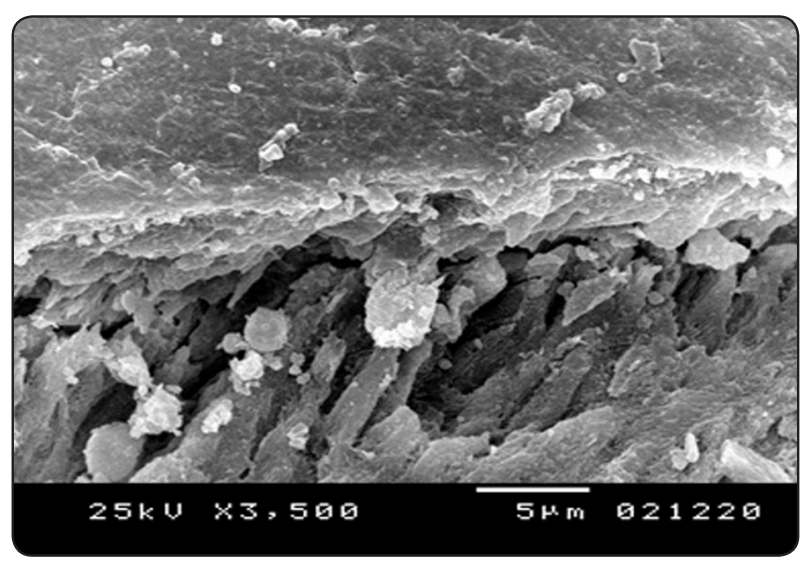

Fig. (3) SEM image, 3500x, showing precipitation of nano hydroxy apatite particles on spattered enamel surface and covered by bonding agent. This group suffered from micro-gap formation at tooth adhesive interface. 


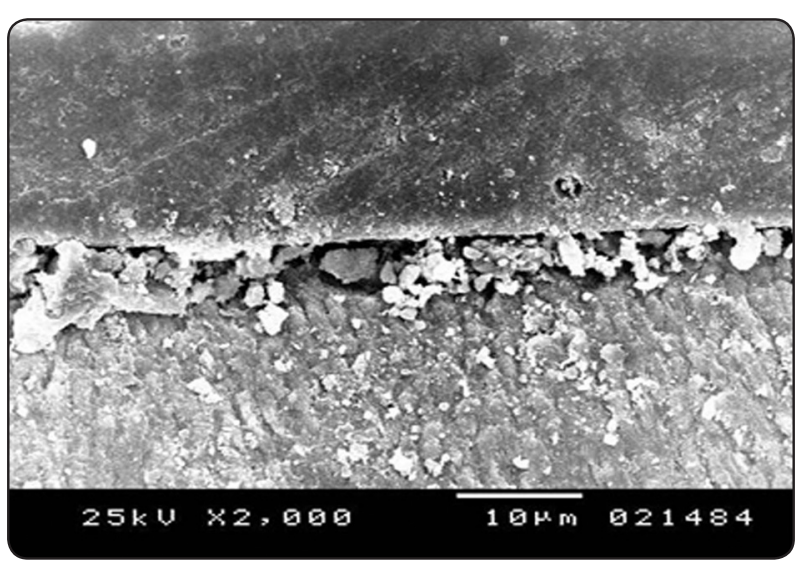

Fig. (4) SEM image, 2000x, showing micro-gap formation at tooth adhesive interface after spattering of nanoparticles.

\section{DISCUSSION}

The selected demineralization model used in this study produced surface features similar to those observed in early enamel carious lesions as the characteristic selective demineralization pattern observed in white spots observed clinically, Figure 2, were the inter-rod enamel region was the most affected ${ }^{(21)}$. Cross section micro-hardness test revealed a mean lesion depth higher than that reported in literature ${ }^{(13,22)}$. This may be attributed to the lower $\mathrm{pH}$ used in this study. Demineralized enamel lesions lost almost $46.9 \%$ of its hardness compared to sound enamel with mean lesion hardness of $202.6 \mathrm{HV}$, these results coincide with results reported in literature ${ }^{(13,23)}$.

Regarding bond strength, direct spattering of nHA on demineralized enamel resulted in reductions in MTBS values and in clustering of the sputtered particles atop of demineralized enamel. Although nHA could be directly adsorbed on demineralized enamel (12) it seems that bond strength was negatively affected due to absence of good contact between resin adhesive and the conditioned enamel substrate, Figure 3 and 4 . Failure pattern in this group tended to be adhesive between resin adhesive and the clustered particles.
The incorporation of $0.2 \%$ wt nano-hydroxyapatite into resin adhesive resulted in improved or maintained bond strength to sound enamel and dentin ${ }^{(17,18,24,25)}$. Unfortunately, no results were reported about the effect of mixing of $4 \% \mathrm{wt}$ nHA with resin adhesives on bond strength. In the present study $4 \%$ wt nHA was the highest concentration that did not negatively affect bond strength and maintained good contact with demineralized enamel. The proposed hypothesis was rejected. Several investigations are needed to study the effect of nano hydroxyapatite on remineralization of early enamel lesions and to study transfer of ions namely calcium and phosphorous between the particles and the enamel surface.

\section{CONCLUSION}

Within the limitations of this study, addition of $4 \%$ wt nano hydroxyapatite to the used self-etch adhesive did not compromise bond strength to demineralized enamel.

\section{REFERENCES}

1. Hediyeh Toutouni, Mohammad-Reza Nokhostin, Bennett Tochukwu Amaechi, Abdol Hamid Zafarmand. The Prevalence of Early Childhood Caries among 24 to 36 Months Old Children of Iran: Using the Novel ICDAS-II Method. Journal of Dentistry, Shiraz University of Medical Sciences 2015;16(4):362-370.

2. Hakan Colak, Coruh T. Dülgergil, Mehmet Dalli,Mehmet Mustafa Hamidi. Early childhood caries update: A review of causes, diagnoses, and treatments. Journal of Natural Science, Biology and Medicine 2013; 4(1): 29-38.

3. Dorozhkin SV. Calcium orthophosphates in dentistry. Journal of Materials Science:Materials in Medicine 2013;24:1335-1363.

4. C. Holmgren, C. Gaucher, N. Decerle-\& S. Doméjean: Minimal intervention dentistry II: part 3. Management of non-cavitated (initial) occlusal caries lesions - non-invasive approaches through remineralisation and therapeutic sealants. British Dental Journal 2014;216,237-243

5. J. Shanti, Arathi Rao. Enamel surface remineralization: Using synthetic nanohydroxyaptite. Contrmporary Clinical Dentistry 2012;.3(4):433-436 
6. Selwitz RH, Ismail AI, Pitts NB. Dental caries. The Lancet 2007;369:51-59.

7. Zero DT. Dentifrices, mouthwashes, and remineralization/ caries arrestment strategies. BMC Oral Health 2006;6:S9.

8. Roza Haghgoo, Mohammad Bagher Rezvani, Mehdi Salehi Zeinabadi. Comparison of Nano- Hydroxyapatite and Sodium Fluoride Mouthrinse for Remineralization of Incipient Carious Lesions. Journal of Dentistry,Tehran University of Medical Sciences 2014;11(4):406-410.

9. Lynch RJM, Smith SR. Remineralization Agents - New and Effective or Just Marketing Hype? Advances in Dental Research 2012;24:63-67.

10. Peter Tschoppe , Daniela L. Zandim , Peter Martus ,Andrej M. Kielbassa. Enamel and dentine remineralization by nano-hydroxyapatite toothpastes. Journal of dentistry 2011; 39:430-437

11. Huang SB, Gao SS, Yu HY. Effect of nano-hydroxyapatite concentration on remineralization of initial enamel lesion in vitro. Biomedical Materials 2009;4:034104.

12. Li L, Pan H, Tao J, Xu X, Mao C, Gu X, et al. Repair of enamel by using hydroxyapatite nanoparticles as the building blocks. Journal of Materials Chemistry 2008; $18: 4079$.

13. Huang S, Gao S, Cheng L, Yu H. Combined effects of nanohydroxyapatite and Galla chinensis on remineralisation of initial enamel lesion in vitro. Journal of dentistry 2010;38:811-819.

14. Tschoppe P, Zandim DL, Martus P, Kielbassa AM. Enamel and dentine remineralization by nano-hydroxyapatite toothpastes. Journal of dentistry 2011;39:430-437.

15. Orsini G, Procaccini M, Manzoli L, Giuliodori F, Lorenzini A, Putignano A.A double-blind randomized-controlled trial comparing the desensitizing efficacy of a new dentifrice containing carbonate/hydroxyapatite nanocrystals and a sodium fluoride/potassium nitrate dentifrice. Journal of Clinical Periodontology 2010;37:510-517
16. Marcelo G., Patricia M., Ana P. A., Paulo M., Bruna F. Toru N., Junji T. Self-Etch adhesive systems: a Literature Review. Brazilian Dental Journal 2015; 26(1): 3-10.

17. Sadat-Shojai M, Atai M, Nodehi A, Khanlar LN. Hydroxyapatite nanorods as novel fillers for improving the properties of dental adhesives: synthesis and application. Dental Materials 2010;26:471-482.

18. Akhavan A, Sodagar A, Mojtahedzadeh F, Sodagar K. Investigating the effect of incorporating nanosilver/ nanohydroxyapatite particles on the shear bond strength of orthodontic adhesives. Acta Odontologica Scandinavica 2013;71:1038-1042.

19. Laura M, Matthias K, Robert C, Helge F B, Bernd K. Shear bond strength of orthodontic brackets to enamel after application of a caries infiltrant. The Angle Orthodontist $2015 ; 85: 654-650$

20. Ten Cate JM, Duijsters PPE. Alternating Demineralization and Remineralization of Artificial Enamel Lesions. Caries Research 1982;16:201-210.

21. Arends J, Jongebloed WIM, ÖGaard B, RÖLla G. SEM and microradiographic investigation of initial enamel caries. European Journal of Oral Sciences 1987;95:193-201.

22. Paris S, Meyer-Lueckel H, Colfen H, Kielbassa AM. Resin infiltration of artificial enamel caries lesions with experimental light curing resins. Dental materials journal 2007;26:582-588.

23. Delbem AC, Cury JA. Effect of application time of APF and $\mathrm{NaF}$ gels on microhardness and fluoride uptake of in vitro enamel caries. American Journal of Dentistry 2002;15:169-172.

24. Leitune VCB, Collares FM, Trommer RM, Andrioli DG, Bergmann CP, Samuel SMW. The addition of nanostructured hydroxyapatite to an experimental adhesive resin. Journal of Dentistry 2013;41:321-327.

25. Vicente Castelo B, Fabrı Mezzomo C, Rafael Mello T, Daniela Guerra A, Carlos Pérez B, Susana Maria W. The addition of nanostructured hydroxyapatite to an experimental adhesive resin Journal of dentistry 2013; 41: 321-327 